FIB-10404-CE

DOI: $10.1007 / \mathrm{s} 11515-016-1404-4$

verso: Afferent DA input to dentate gyrus

recto: Charlotte M. Ermine et al.

RESEARCH ARTICLE

\title{
Combined immunohistochemical and retrograde tracing reveals little evidence of innervation of the rat dentate gyrus by midbrain dopamine neurons
}

\author{
Charlotte M. Ermine $(\bowtie)^{1}$, Jordan L. Wright ${ }^{1,2}$, Clare L. Parish ${ }^{1}$, Davor Stanic ${ }^{1}$, \\ Lachlan H. Thompson ${ }^{1}$ \\ ${ }^{I}$ The Florey Institute for Neuroscience and Mental Health, The University of Melbourne, Parkville, Victoria 3010, \\ Australia \\ ${ }^{2}$ Current address: Wolfson Institute for Biomedical Research, University College London, Gower Street, London \\ WCIE 6BT, UK
}

Received March 15, 2016; accepted May 20, 2016

Correspondence: Charlotte M. Ermine

E-mail: charlotte.ermine@florey.edu.au

CHigher Education Press and Springer-Verlag Berlin Heidelberg 2016

\begin{abstract}
Although the functional neuroanatomy of the midbrain dopamine (mDA) system has been well characterized, the literature regarding its capacity to innervate the hippocampal formation has been inconsistent. The lack of expression of definitive markers for dopaminergic fibers, such as the dopamine transporter, in the hippocampus has complicated studies in this area. Here we have used immunohistochemical techniques to characterize the tyrosine hydroxylase expressing fiber network in the rat hippocampus, combined with retrograde tracing from the dentate gyrus to assess the capacity for afferent innervation by mDA neurons. The results indicate that virtually all tyrosine hydroxylase fibers throughout the hippocampus are of a noradrenergic phenotype, while the overlying cortex contains both dopaminergic and noradrenergic fiber networks. Furthermore, retrograde tracing from the dentate gyrus robustly labels tyrosine hydroxylase-immunoreactive noradrenergic neurons in the locus coeruleus but not mDA neurons.
\end{abstract}

Keywords noradrenaline, hippocampus, connectivity, DAT, neurogenesis

\section{Introduction}

Recent studies have reported that loss of midbrain dopamine (mDA) neurons in the rodent brain results in a reduction in hippocampal cell proliferation and neurogenesis (Suzuki et al., 2010), and can also impair cognitive function (Wisman et al., 2008). Interestingly, the rate of hippocampal neurogenesis has been shown to decline sharply with age (Ben Abdallah et al., 2010; Spalding et al., 2013)with a concomitant cognitive decline, and this is thought to be part of the pathophysiological basis of age-related dementia(Drapeau et al., 2003; Small et al., 2011; Seib et al., 2013). It might also be a point of convergence for other pathological changes and cognitive decline in neurodegenerative disease. For example, in Parkinson's disease (PD), in addition to the well-characterized motor dysfunction resulting from the loss of mDA neurons, a subset of patients develops dementia - particularly during the end-stages of the disease(Levy et al., 2002; Emre, 2003). 
Studies investigating the role of dopaminergic signaling in adult neurogenesis have primarily focused on the sub-ventricular zone (SVZ). The SVZ is in close proximity to the rich dopaminergic innervation of the striatum and removal of the dopamine (DA) neurons has been shown to reduce precursor cell proliferation in the SVZ in PD patients and animal models of dopamine depletion (Baker et al., 2004; Höglinger et al., 2004; Freundlieb et al., 2006) - reviewed in (Regensburger et al., 2014). The potential impact on hippocampal neurogenesis in the subgranular zone of the dentate gyrus is less well understood and it is unclear whether the dentate gyrus receives any significant projections from the mDA projection system.

The mDA system is one of the most well characterized projection systems in the rodent brain (Björklund and Dunnett, 2007). However, there has been lack of consensus in the literature regarding its capacity to innervate the hippocampus. Early studies highlighted very low levels of DA in the hippocampus compared with other well-characterized target structures, such as the striatum (Bjorklund, 1978), and led to the view that the hippocampus did not receive an afferent dopaminergic input. In contrast, subsequent work reported that hippocampal DA levels were sensitive to selective lesions of the mDA system (Bischoff et al., 1979; Scatton et al., 1980). Tract tracing studies have reported either the presence (Swanson, 1982; Gasbarri et al., 1994) or absence (Reymann et al., 1983; Pohle et al., 1984) of retrograde labeled mDA neurons after injections of tracer into different hippocampal regions. Further complicating the scenario is the presence of DA receptors in the hippocampus(Dubois et al., 1986), but absence of the dopamine transporter (DAT) on putative pre-synaptic DA fibers, leading to the idea that the lack of DAT expression is a specialized feature of the DA innervation of the hippocampus. On the other hand, there has also been recent work suggesting that the noradrenergic (NA) hippocampal innervation from the locus coeruleus (LC) acts as the primary, and perhaps exclusive, source of hippocampal DA signaling (Borgkvist et al., 2012; Smith and Greene, 2012).

Here we sought to investigate the presence of midbrain-derived dopaminergic projections in the septal hippocampus through microinjection of the retrograde tracer fluorogold (FG) into the dentate gyrus. As a basis for comparison, we also describe the retrograde labeling of NA neurons in the LC, which are known to densely innervate the hippocampus, particularly the sub-granular region of the dentate gyrus.

\section{Materials and methods}

\section{Animals}

Adult female Sprague Dawley rats were used for retrograde tracing studies, with all experiments approved by the Florey Institute for Neuroscience and Mental Health animal ethics committee. All animals were housed in individually ventilated cages under a $12 \mathrm{~h}$ light/dark cycle with ad libitum access to food and water.

\section{Surgical procedures and tissue preparation}

Rats were deeply anesthetized with isoflurane (induction at 5\%, maintenance at $2 \%$ ) and placed in a stereotaxic frame (Kopf, Germany). A small burr-hole was drilled in the skull $3.8 \mathrm{~mm}$ caudal and $1.8 \mathrm{~mm}$ lateral to bregma, and $0.1 \mu \mathrm{L}$ of $2 \%$ FG (Fluorochrome, Denver, CO, USA) was microinjected into the hilus of the dentate gyrus using a 'Neuros' Hamilton syringe over a period of $1 \mathrm{~min}$. The cannula was left in place a further $5 \mathrm{~min}$ to limit reflux along the injection tract. After 5-7 days the animals were killed by lethal injection of pentobarbitone $(100 \mathrm{mg} / \mathrm{kg}$; Virbac, Peakhurst, Australia) and were transcardially perfused with Tyrode solution $(1 \mathrm{~mL} / 1 \mathrm{~g})$, followed by $4 \%$ paraformaldehyde in $0.4 \mathrm{M}$ phosphate buffer with $0.2 \%$ picric acid. The brain was then collected and post-fixed for $2 \mathrm{~h}$ in the fixation media followed by cryoprotection in $20 \%$ sucrose PBS solution for 1-2 days. All brains were frozen on dry ice and sectioned in the coronal plane in to $40 \mu \mathrm{m}$ sections using a freezing-microtome (Leica, Wetzlar, Germany). 


\section{Immunohistochemistry}

Free-floating immunohistochemistry was performed on a 1:12 series as previously described (Thompson, 2005). Briefly the tissue sections were incubated overnight with primary antibodies diluted in blocking solution (5\% donkey serum and $0.5 \%$ Triton X in PBS) and subsequently for 2 $\mathrm{h}$ with fluorophore-conjugated secondary-antibodies diluted in the same blocking solution. The sections were mounted on glass slides and coverslipped using DAKO mounting media (USA). The primary antibodies used were: mouse anti-tyrosine hydroxylase (TH, Immunostar; 22941, 1:1000), mouse anti-dopamine- $\beta$-hydroxylase (DBH, Millipore; MAB308, 1:5000); goat anticholine acetyltransferase (ChAT, Millipore; MAB144, 1:100); rabbitanti- $\gamma$ aminobutyric acid (GABA, Sigma; A2052, 1:1000); rabbitanti-5-hydroxytryptamine (5-HT, Immunostar, 20080, 1:5000); rabbitanti-fluorogold (FG, Millipore; MAB153 1:1000); rabbit anti-Prox1 (Millipore, ABN278, 1:2000);goatanti-doublecortin (DCX, Santa Cruz sc-8066, 1:1000) ratanti-dopamine transporter (DAT, Millipore, MAB369; 1:3000).The secondary antibodies used were conjugated to Dylight Fluorophores 488, 549, or 647 anti-mouse, anti-rabbit, anti-sheep, or anti-goat (Jackson ImmunoResearch, West Grove, PA, USA; 1:400). For amplified detection of DAT, a horseradish peroxidase (HRP)-conjugated secondary antibody was used (swine anti-rat, Jackson ImmunoResearch, West Grove, PA, USA, 1:500) followed by incubation with biotinyl tyramidefluoroscein for 10 min using a commercial kit (TSA + , NEN Life Science Products, Inc., Boston, MA) as previously described (Sui et al., 2012).

\section{Analysis}

All microscopic analysis was performed using a Zeiss inverted microscope (AxioObserver.Z1) or Zeiss Meta confocal microscope (LSM 780). The presence of double-labeled TH and FGimmunoreactive cells within the midbrain or LC was verified using confocal microscopy. Schematic overviews of the distribution of FGlabeled cells in these areas were generated using Inkscape software. Cell counts were performed twice to ensure accuracy.

\section{Results}

\section{Immunohistochemical detection of dopaminergic and noradrenergic fibers in the hippocampus}

The hippocampus of the adult rat brain is richly innervated by tyrosine hydroxylase (TH)expressing fibers (Fig. $1 \mathrm{~A}-\mathrm{E}$ ). This includes a dense TH + fiber network in and around the dentate gyrus, particularly the sub-granular layer (SGL) where newborn doublecortin-expressing neurons are generated before migrating into the overlying granule layer as Prox $1+$ interneurons (Fig. 1). Immunohistochemistry for dopamine-beta-hydroxylase (DBH), expressed exclusively in NA fibers, revealed a similarly dense innervation of the SGL and surrounding dentate gyrus (Fig. 1 F-J), while DAT labeling did not reveal the presence of any DAT fibers (Fig. $1 \mathrm{~K}-\mathrm{O}$ ) throughout the hippocampus. Double-labeling for TH and DBH revealed a complete overlap of expression within the dentate gyrus (Fig. $1 \mathrm{P}-\mathrm{S}$ ). Careful inspection via confocal microscopy showed good penetration of both antibodies throughout the $40 \mu \mathrm{m}$ tissue sections and we were unable to find examples of $\mathrm{TH}+$ fibers that were not immunoreactive against $\mathrm{DBH}$. This was also the case when looking at other areas receiving prominent $\mathrm{TH}+$ innervation, including the molecular layer of the dentate gyrus and CA3 (Fig. 2 A, B, C), and, indeed, throughout the entire hippocampus (not shown). However, in the overlying cingulate cortex (CC), discrete patches of $\mathrm{TH}+/ \mathrm{DAT}+$ fibers were clearly distinguishable among the more diffuse network of TH+/DBH+ fibers (Fig. 2 A, D). 

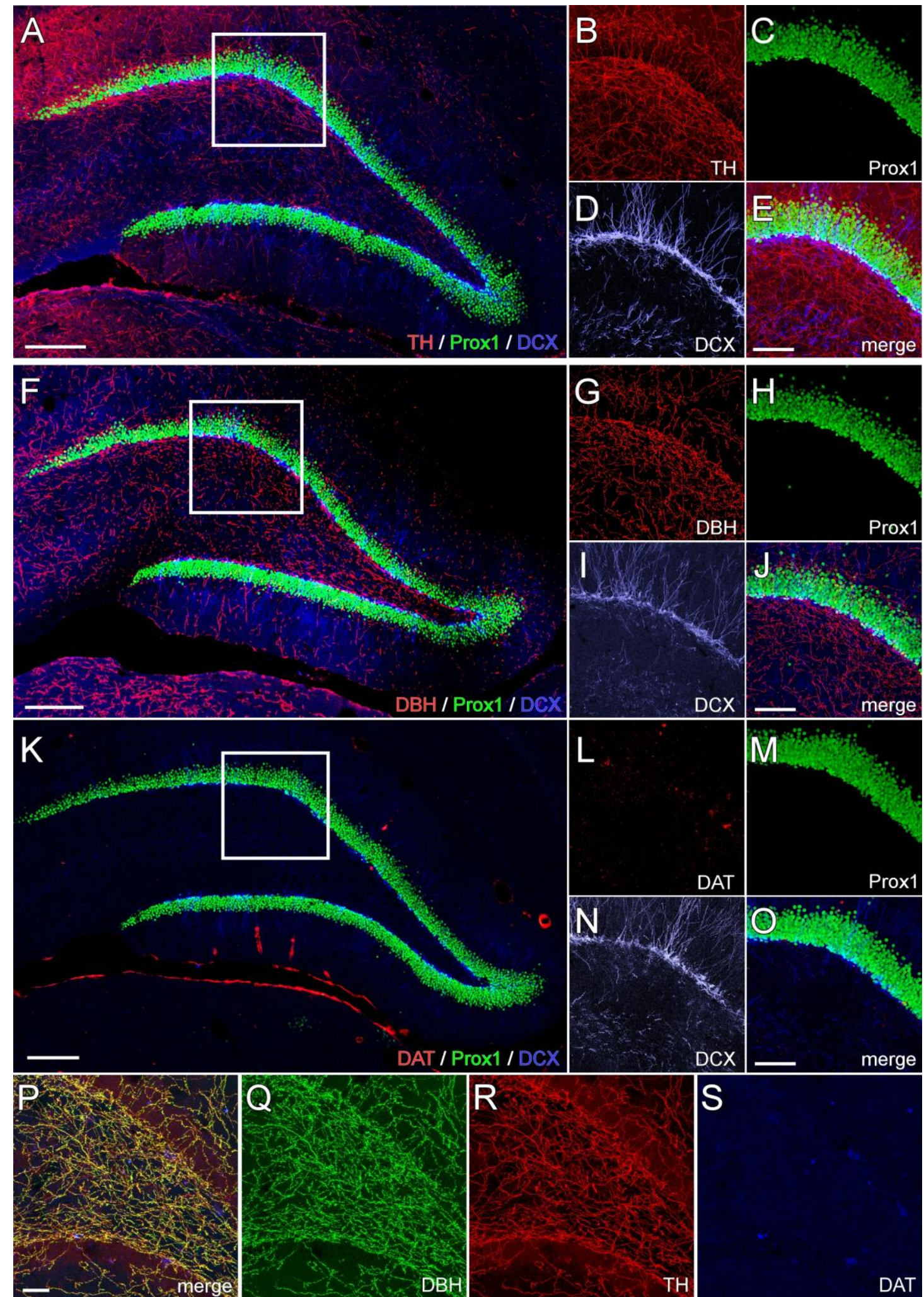

Figure 1 Immunohistochemical detection of dopamine and noradrenaline fibers in the rat dentate gyrus. The dentate gyrus contains a fiber network that can be labeled with antibodies against tyrosine hydroxylase (A) or dopamine- $\beta$-hydroxylase (F) but not the dopamine transporter (K). Enlargements of the boxed areas in A and F show that TH (B-E) and DBH (G-J)fiber innervation is particularly dense in and around the sub-granular and granular layers, identified by doublecortin and Prox 1 respectively, while there is a complete absence of DAT expression ( $\mathrm{L}-\mathrm{O})$. Triple-labeling for TH, DBH, and DAT (P-S) shows that all TH+ fibers express DBH, while none co-label with DAT. Scale bars:A, F, and K, $200 \mu \mathrm{m}$ and B-E, G-J, L-O, and P-S, $50 \mu \mathrm{m}$. Abbreviations: DAT, Dopamine transporter; DBH, Dopamine-beta-hydroxylase; DCX, Doublecortin; Prox1, Prospero homeobox1; TH, Tyrosine hydroxylase. 

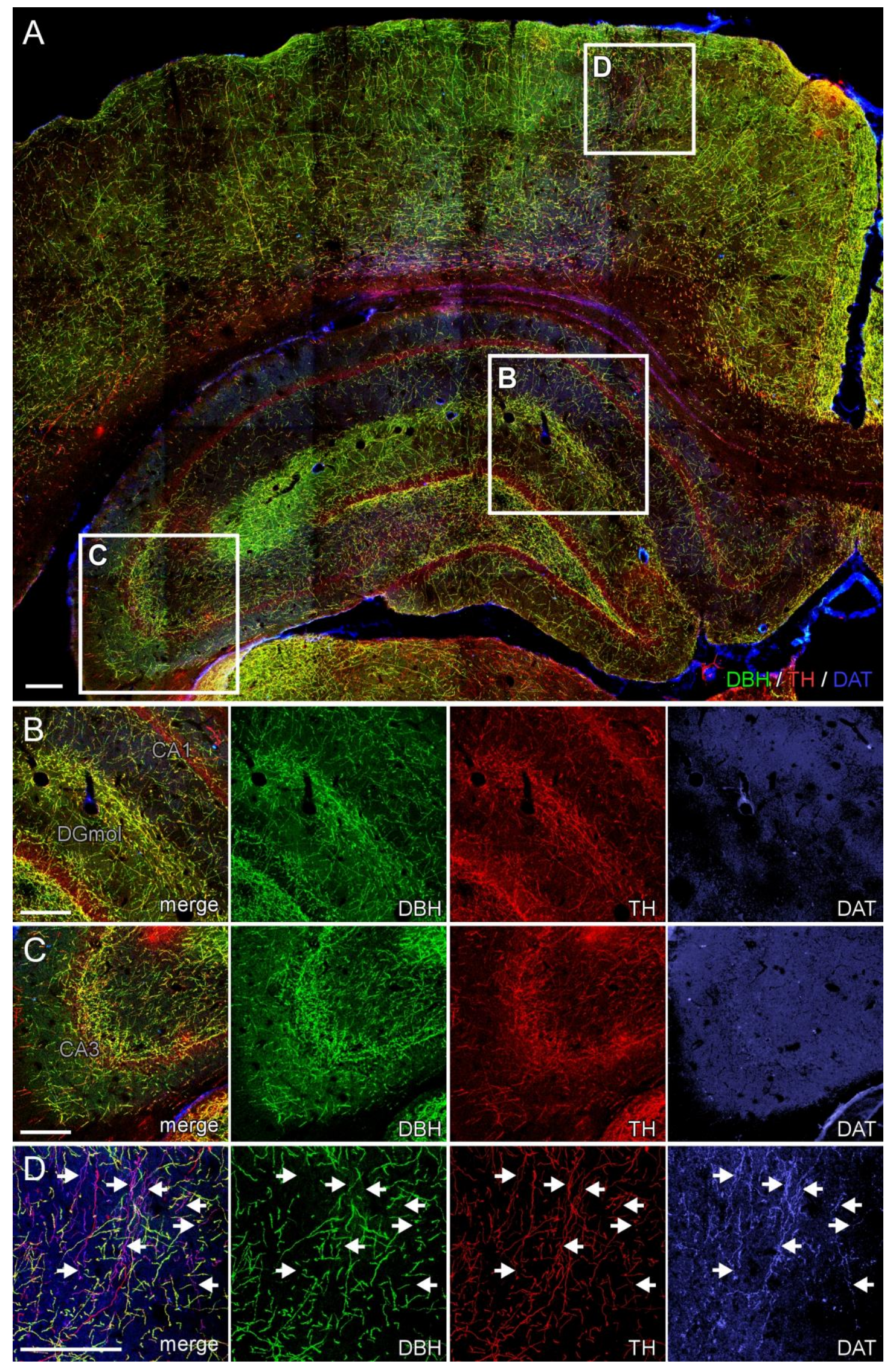

Figure 2 Overview of dopamine and noradrenergic fibers in the septal hippocampus and overlying cortex. A) Photomontage of immunohistochemical detection of TH, DBH, and DAT in the rat hippocampus and cortex. Boxed areas are shown as enlarged views of hippocampal areas with prominent TH fiberlabeling, including the sub-granular zone and molecular layer of the dentate gyrus (B) and CA3 (C) and also the overlying cortex (D) 
where TH+/DA+ fibers (arrows) were intermingled with TH/DBH fibers. Scale bars: A, $200 \mu \mathrm{m}$ and B-D, $50 \mu \mathrm{m}$. Abbreviations: CA1, Cornus Ammonis 1; CA3, Cornus Ammonis 3; DGmol, Dentate gyrus molecular layer.

\section{Injection of fluorogold into the dentate gyrus retrogradely labels neurons in the ventral midbrain and the locus coeruleus}

Detection of FG, using immunohistochemistry, allowed for verification of the location and extent of delivery to the dentate gyrus. In 10 animals used for the analysis of retrograde labeling patterns, FG immunoreactivity was confined to a discrete area on the medial dentate gyrus in the septal hippocampus, including the ventral and dorsal blades, and adjacent hilus, and in some animals, additional labeling of the overlying molecular layer and CA1 area (Fig. 3A). FG was retrogradely transported to neurons in a number of nuclei throughout the brain, including those with wellcharacterized projections to the hippocampus such as the cholinergic neurons in the septum and nucleus basalis and serotonergic neurons of the raphe nucleus (Supplemental Fig. 1).
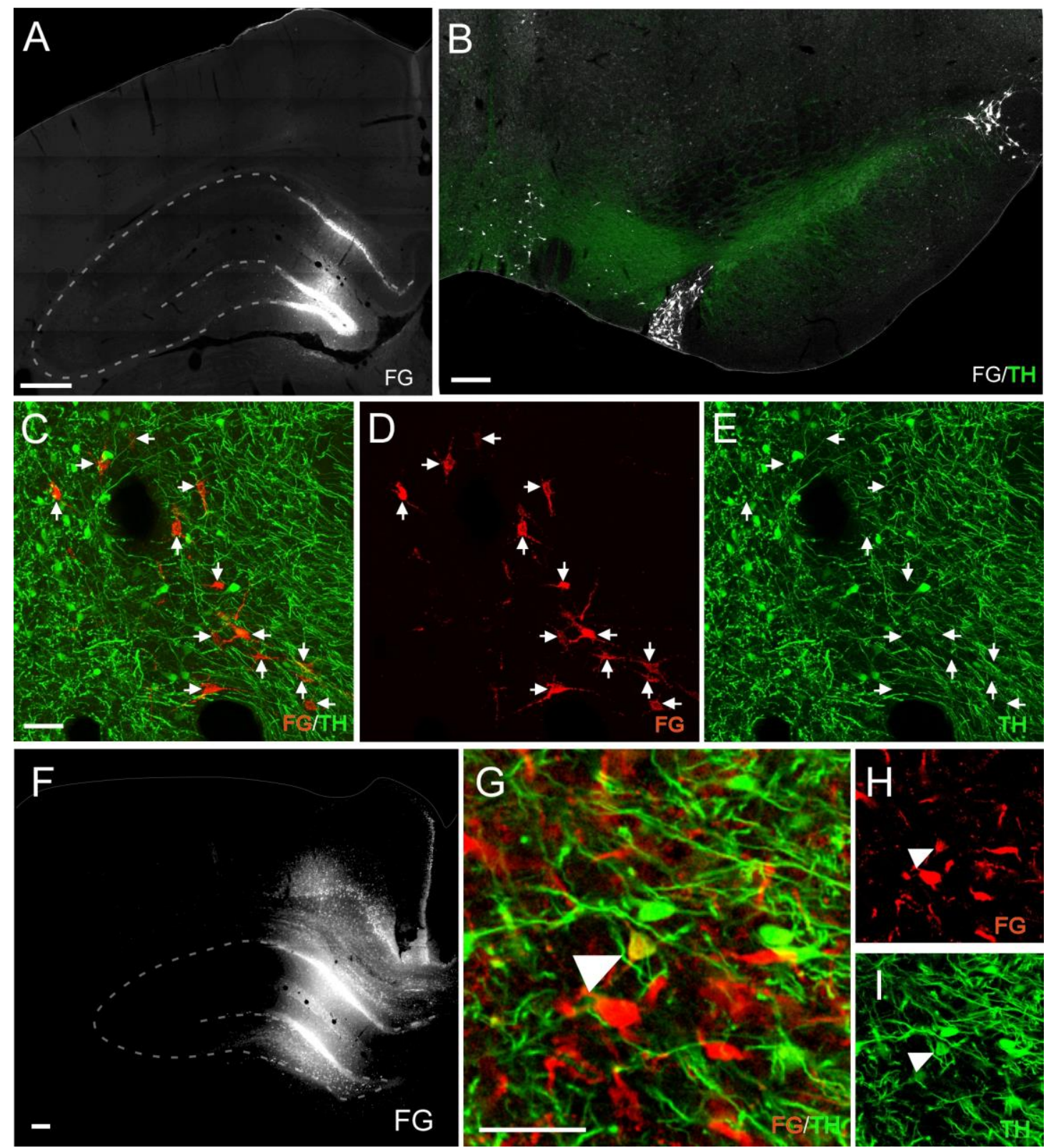

Figure 3 Retrograde labeling of midbrain neurons 5-7 days following injection of fluorogold into the dentate gyrus. Immunolabeling of fluorogold in a representative animal at the injection site (A) showing distribution in the dentate gyrus and some labeling in the overlying molecular layer and CA1, and in the midbrain(B), showing 
retrogradely labeled neurons with TH labeling (green) shown for anatomical reference. (C-E) Merged image and individual color channels showing fluorogold-labeled cells (arrows) interspersed among, but not overlapping with TH+ DA neurons in the VTA. (F) Representative fluorogold labeling at the injection site in one of two animals where fluorogold labeling was found along the injection tract in the cortex.(G-I) In these animals a total of three fluorogold-labeled TH+ DA neurons were identified in the midbrain. Scale bars:A, B, F, $200 \mu \mathrm{m}$; C-E and G-I, 50 $\mu \mathrm{m}$. Abbreviations: FG, Fluorogold; TH, Tyrosine hydroxylase.

In the ventral midbrain, FG+ neurons were consistently found distributed in and around midline nuclei, including the paranigral, parabrachial pigmented, interfascicular, interpeduncular, and rostral linear raphe nuclei, and throughout the ventral tegmental area (VTA) (Fig. 3B and 4A).In all animals, there was prominent labeling of cells closely associated with the optic tract, including the medial and lateral terminal nuclei of the accessory optic tract (Fig. 3B).

Close inspection using confocal microscopy of midbrain sections labeled for TH in order to identify mDA neurons, showed that, overwhelmingly, the FG+ neurons did not co-label with TH (Fig. 3 C-E). However, we did identify a total of three DA neurons across two of the 10 animals that were unambiguously co-labeled with TH (Fig. 3 G-I). Notably, in both of these animals detection of FG showed labeling in the cortex overlying the dentate gyrus target site (Fig. 3F). A total of $605 \mathrm{FG}+$ cells were identified through the examination of three coronal sections spanning most of the rostro-caudal extent of the territory covered by the A9 and A10 DA cell groups in the 10 animals. Figure 4 shows the cumulative spatial distribution of these FG cells (red circles), and the three FG+/TH+ cells (yellow circles), in the representative sections. Retrograde labeling was more prominent in the rostral sections and approximately $8 \%$ of the FG+ cells were located in the midbrain contralateral to the injected hippocampus. Further immunohistochemical characterization showed that some of the FG+ cells had a GABAergic phenotype(Fig. 4 BD).Poor penetration of the GABA antibody prevented a conclusive assessment of the GABAphenotype across the majority of FG+ cells at a more quantitative level.
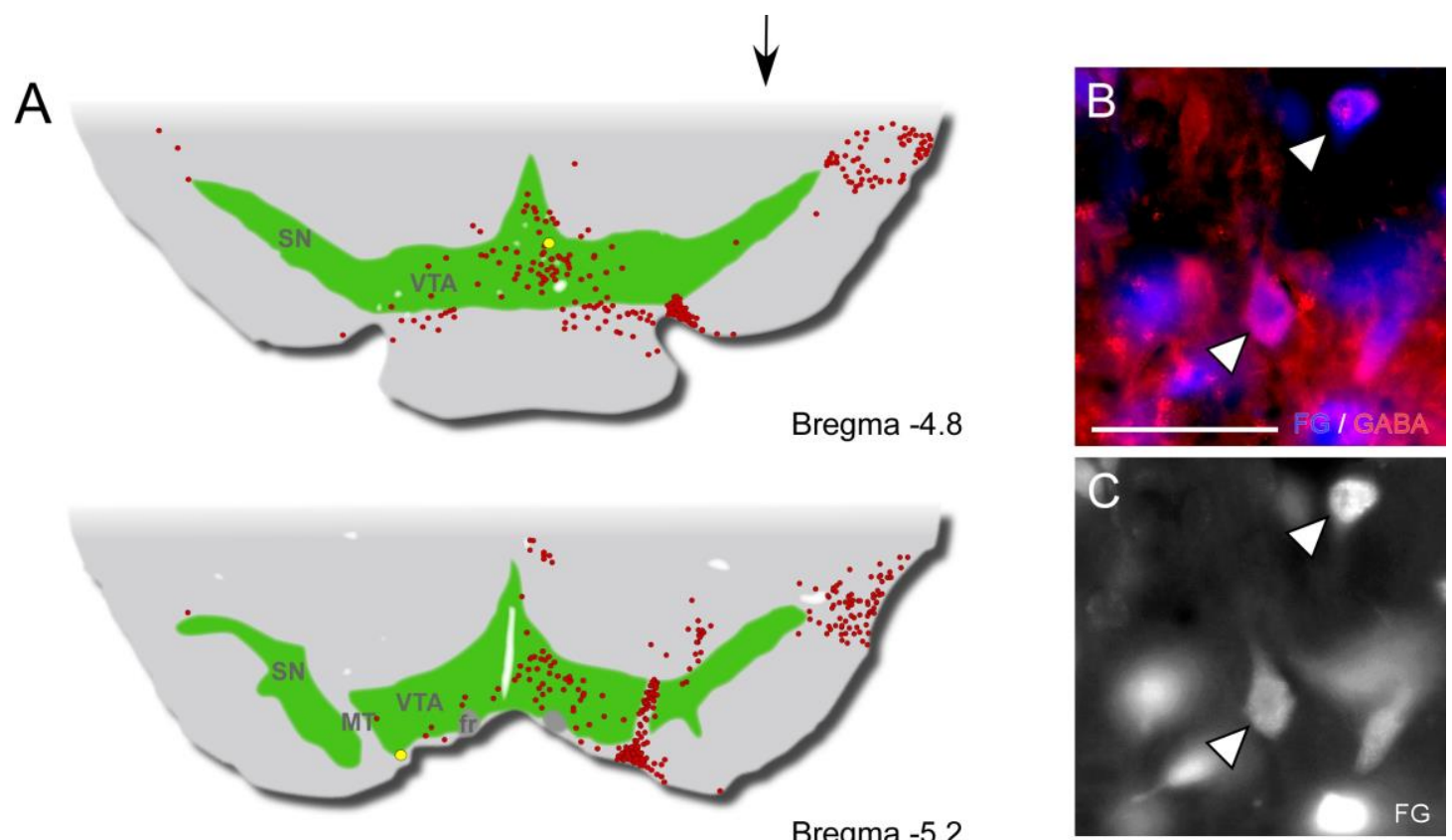

Bregma -5.2
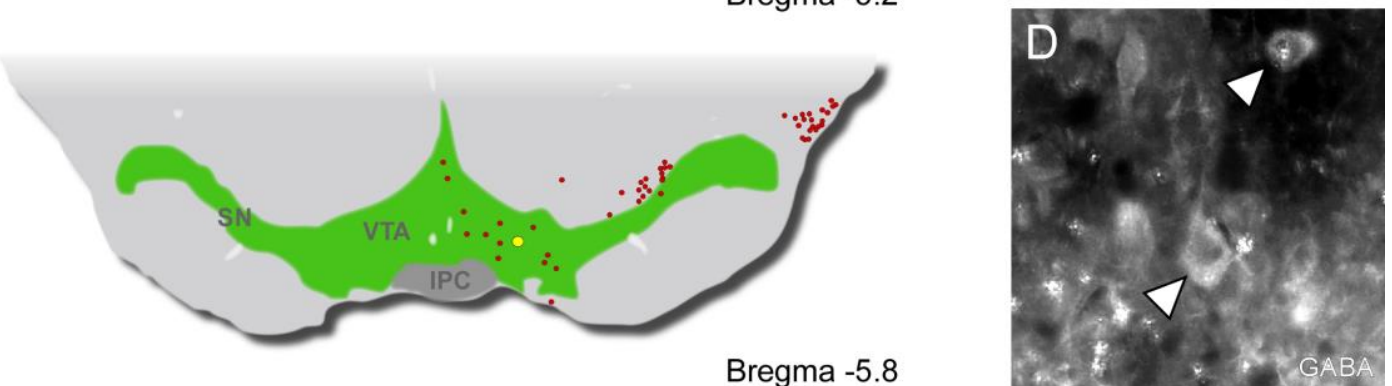

Bregma -5.8 
Figure 4 Cumulative spatial representation of fluorogold labeling in the midbrain of 10 animals 5-7 days after injection of fluorogold into the dentate gyrus. (A) Schematic overview of three representative coronal levels spanning the A9-A10 cell groups illustrating the location of all 605 fluorogold-labeled cells, indicated as red circles for $\mathrm{FG}+/ \mathrm{TH}$ - cells and yellow circles for $\mathrm{FG}+/ \mathrm{TH}+$ cells. The black arrow represents the ipsilateral side injected with fluorogold. (B-D) Co-labeling for GABA showed that some of the FG+ neurons innervating the hippocampus were GABAergic projection neurons. Scale bars: B-D, $50 \mu \mathrm{m}$. Abbreviations: FG, Fluorogold; fr, fasciculus retroflexus; GABA, $\gamma$ aminobutyric acid; IPC, Interpeduncular nucleus central; MT, Medial terminal nucleus accessory optic tract; SNr, Substantia nigra reticular; VTA, Ventral tegmental area.

Inspection of the LC, as identified by $\mathrm{TH}+$ cell bodies, showed the presence of FG+ cells in all animals that received hippocampal FG injections (Fig. 5 A-C). In all cases, cells were found in the ipsilateral LC, with a smaller contribution of cells found in the contralateral LC (Fig. 5 D). Cells were also identified bilaterally in the adjacent central gray pons (PCG). Figure $5 \mathrm{D}$ illustrates the cumulative distribution of FG+ cells in 10 animals across three coronal sections spanning the rostro-caudal extent of the LC. Confocal analysis showed that the vast majority of the FG+ cells were NA neurons based on the co-expression of TH (Fig. 5 A-C).
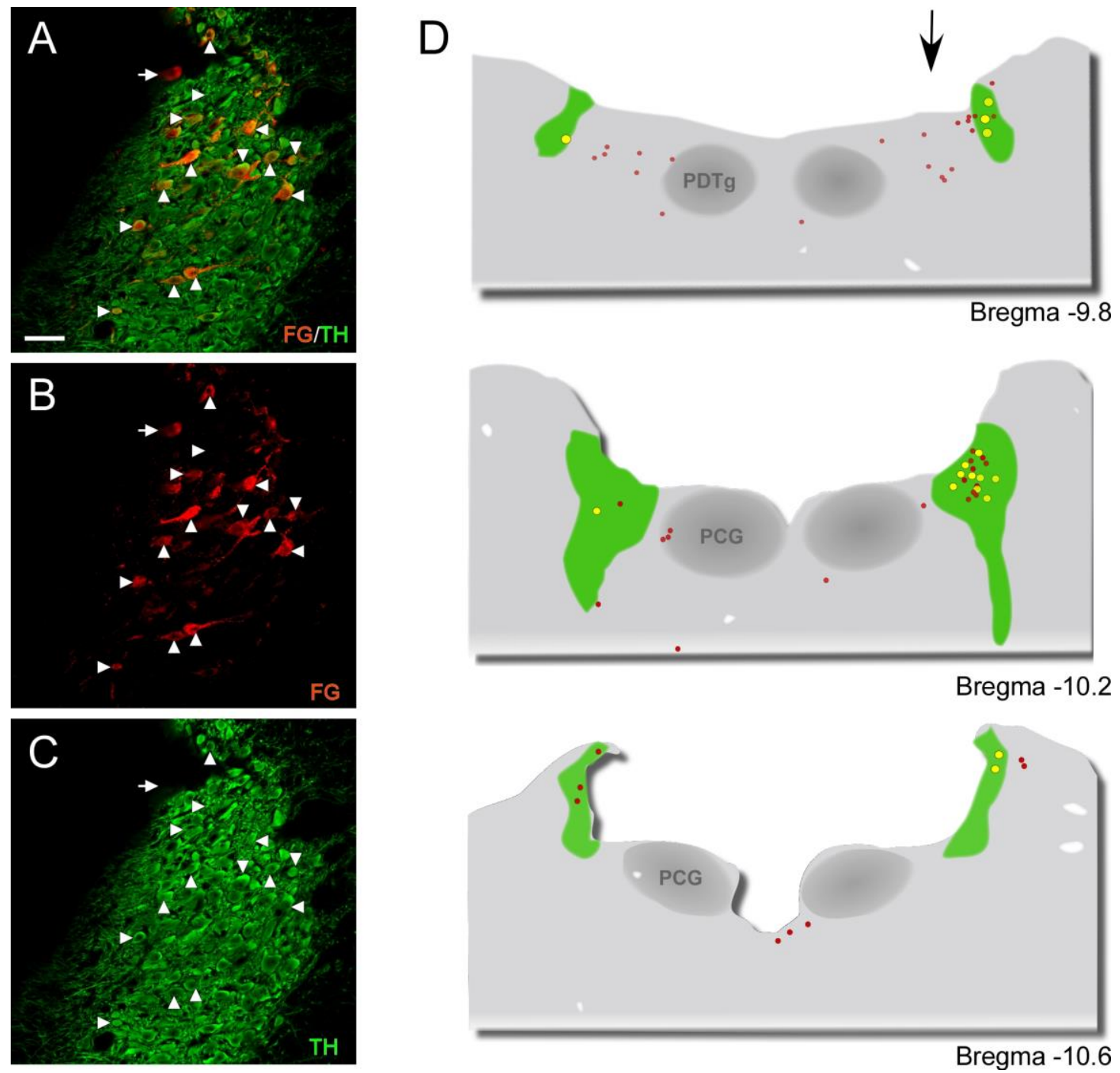

Figure 5 Retrograde labeling of noradrenergic neurons in the locus coeruleus 5-7 days after injection of fluorogold into the dentate gyrus. (A-C) Immunolabeling of fluorogold and TH showed extensive overlap in the locus coeruleus (arrowheads) as well as a minor population of FG+/TH- cells (arrows). (D)Schematic overview of three representative coronal levels spanning the locus coeruleus where the location of fluorogold labeled cells are indicated as red circles for FG+/TH- cells and yellow circles for FG+/TH+ cells. The black arrow represents the ipsilateral side injected with fluorogold. Scale bars: A-C, $200 \mu \mathrm{m}$. Abbreviations: FG, fluorogold; TH, tyrosine hydroxylase; PCG, pontine central gray; PDTg, posterodorsal tegmental nucleus. 


\section{Discussion}

These results indicate that the adult rat dentate gyrus receives very little, if any, afferent input from mDA neurons. Tyramide-based amplification allowed for the robust detection of DAT+ fibers in the brain with a resolution of single fibers, as seen in the CC. Although it has previously been suggested that DA fibers in the hippocampus do not express DAT, detailed confocal analysis showed that $\mathrm{TH}+$ fibers throughout the hippocampal formation universally co-labeled with $\mathrm{DBH}$, indicating they were derived from NA projections from the LC. Therefore, it is unlikely that the hippocampus contains DA terminals that do not express DAT.

The absence of definitive histological markers for DA terminals in the hippocampus has necessitated indirect means to infer their presence. For example, the presence of hippocampal DA receptors (Dubois et al., 1986), biochemical detection of low levels of hippocampal DA that can be partly reduced by chemical or electrolytic lesioning of the midbrain (Bischoff et al., 1979; Scatton et al., 1980), and the persistence of DA and DOPAC levels (Bischoff et al., 1979; Scatton et al., 1980) or TH+ fibers (Verney et al., 1985)in rats with lesions of the NA system have all been interpreted as evidence for a hippocampal DA terminal network (for review see (Gasbarri et al., 1997)). However, there are caveats to these interpretations. For example, lesioning of the NA system using 6-hydroxydopamine (6OHDA) or N-(2-chloroethyl)-N-ethyl-2-bromobenzylamine (DSP4) are often incomplete, as was the case in the study by Verney et al. (1985), and thus one cannot unequivocally exclude the possibility that the remaining TH+ fibers or DA metabolites originate from spared NA neurons. Similarly, 6OHDA or electrolytic lesioning of the $\mathrm{mDA}$ system can also damage NA neurons. In the study by (Scatton et al., 1980), lesioning of the NA system produced a partial reduction in hippocampal DA content, that was further reduced by electrolytic lesioning targeted at the VTA. It cannot be completely excluded that the severity of an initially incomplete NA lesion is increased by subsequent electrolytic damage to NA axons passing through the midbrain.

Interestingly, studies performed more recently have provided convincing evidence that the NA terminal network serves as the source of DA signaling in the hippocampus. Microdialysis studies measuring DA levels in the hippocampus have shown that DA is cleared by the NA transporter and that antagonism of NA pre-synaptic $(\alpha 2)$ receptors, but not $\mathrm{DA} \mathrm{D}_{2}$ autoreceptors, increased hippocampal DA (Borgkvist et al., 2012). Similarly, (Smith and Greene, 2012) have reported that amphetamine-induced stimulation of DA receptors in the CA1 field is not affected by pharmacological blockade of DAT to prevent amphetamine uptake into DA terminals, or by selective inhibition of VTA input through siRNA knockdown of TH, but is inhibited by blockade of the NA transporter or knockdown of TH in the LC. These findings highlight the important point that a lack of a direct midbrain dopaminergic projection to the hippocampus does not preclude a role for dopamine in modulating hippocampal activity and provide an interesting point of consideration for the neuroanatomical basis of changes in hippocampal dopamine levels in experimental work in rodents (e.g., Kwon et al., 2008). Additionally, it is important to recognize that the lack of a direct projection does not preclude mDA modulation of hippocampal activity through indirect, multi-synaptic pathways. For example, mDA neurons in the VTA are known to innervate the locus coeruleus (for review see (Samuels and Szabadi, 2008)) and thus modulation of hippocampal neuronal firing through activation of the VTA (Rosen et al., 2015) may well occur through this pathway.

Another important approach for characterizing the afferent input to the hippocampus has been through retrograde tracing studies. Early studies in this area confirmed the presence of hippocampal projections originating in the midbrain, but not their neurochemical identity (Meibach and Siegel, 1977; Schwab et al., 1978; Simon et al., 1979; Wyss et al., 1979). These studies showed similar patterns of cell labeling to those reported here, with the majority of labeled cells contained within the VTA and adjacent nuclei, close to the midline. Later studies combined retrograde labeling with fluorescence immunohistochemistry in order to further identify these neurons. Studies by (Reymann et al., 1983; Pohle et al., 1984) report that 'not one neuron' identified as containing biogenic amine in the A9-A10 cell groups was retrogradely labeled, while other studies report 6\%-18\% are dopaminergic (Swanson 1982; Gasbarri et al., 1994). Here we report that following FG injection into the dentate gyrus of the septal hippocampus, only three of $605 \mathrm{FG}+$ cells in the ventral midbrain $(0.5 \%)$ were $\mathrm{TH}+$. Notably, these cells were found in two 
animals with some reflux of FG into the overlying cortex, where, unlike the hippocampus, we noted the presence of DAT $+/ \mathrm{TH}+$ fibers. Given that retrograde labeling studies targeting the hippocampus necessitates that the injection cannula passes through the cortex, one cannot completely exclude 'off-target' labeling of neurons projecting to the cortex. Gasbarri et al.(1994) reported a greater proportion of DA neurons when retrogradely labeling from the temporal (15\%$18 \%$ ) compared with septal (10\%-12\%) hippocampus. Here, we limited the retrograde tracing studies to the septal dentate gyrus. We found the absence of DAT and the complete overlap between DBH and TH to be consistent throughout all aspects of the hippocampus. On this basis, we would not predict a greater propensity for labeling mDA neurons when injecting the tracer into other hippocampal regions. Co-labeling with markers for other neurotransmitter phenotypes showed at least some of the midbrain hippocampal projections to be GABA-containing neurons. Although it is known that the VTA consists of up to $30 \%$ GABA neurons, including interneurons, as well as projections to areas such as cortex and nucleus accumbens ((Carr and Sesack, 2000); for review see (Creed et al., 2014)), projections to the hippocampus have not been well characterized. To obtain additional detailed information on the identity of the FG+ cells, including the quantity and topographical distribution of specific phenotypes, further investigation is certainly warranted.

FG injection into the dentate gyrus also retrogradely labeled TH+ neurons in the dorsal segment of the LC, both ipsilateral and, to a lesser extent, contralateral to the injection. This is consistent with retrograde tracing studies in both rats (Loy et al., 1980) and monkeys (Amaral and Cowan, 1980) showing that NA neurons project bilaterally to the hippocampus. Work by (Loughlin et al., 1986)also showed that projections to the hippocampus are located in the dorsal segment of the LC, while the ventral LC contains neurons projecting to the spinal cord. We did not set out to perform a detailed characterization of the NA innervation of the hippocampus, but rather describe the results from retrograde labeling as a basis for comparison to the labeling in the midbrain.

Compared with the mDA system, the functional neuroanatomy of afferent input to the hippocampus from NA neurons in the LC has been relatively more straightforward to characterize - for reviews see (Harley, 2007; Samuels and Szabadi, 2008; Szabadi, 2013). The development of new tools, such as viral-genetic tracing approaches (Schwarz et al., 2015), has allowed for even further refinement of our understanding of central NA connectivity and their application may help to further characterize and clarify connectivity of other systems, including the mDA system.

In summary, using immunohistochemical and retrograde tracing techniques combined with confocal microscopy, we have found that the $\mathrm{TH}+$ fiber innervation of the rat dentate gyrus is overwhelmingly, and perhaps exclusively, accounted for by NA projections from the LC. mDA neurons appear to provide little, if any, afferent input to the hippocampus, particularly the dentate gyrus. This has implications for how we may interpret data from studies that look at the impact of mDA loss at the level of the hippocampus. For example, reduced neurogenesis (Suzuki et al., 2010) or deficits in cognitive function (Wisman et al., 2008) after 6OHDA lesioning may reflect mechanisms that are more indirect than loss of a direct mDA-hippocampus pathway - i.e., changes in hippocampal signaling at a broader systems level involving dopaminergic innervation of other nuclei that feed forward to the hippocampus. Both the nucleus accumbens and amygdala may be interesting targets to investigate further in this context. Both receive innervation from $\mathrm{mDA}$ neurons and both structures are involved in associative learning tasks through connectivity with the hippocampus (Ito et al., 2008; Sara, 2009; Broussard et al., 2016).

\begin{abstract}
Abbreviations
5-HT, 5-hydroxytryptamine; 6OHDA, 6-hydroxydopamine; CC, Cingulate cortex; ChAT, Choline acetyltransferase; DA, dopamine; DAT, dopamine transporter; DBH, dopamine-b-hydroxylase; DCX, doublecortin; DSP4, N-(2-chloroethyl)-N-ethyl-2-bromobenzylamine; FG, fluorogold; GABA, $\gamma$ aminobutyric acid; HRP, horseradish peroxidase; LC, Locus coeruleus; mDA, midbrain dopamine; NA, noradrenaline; PCG, Pontine central gray; PD, Parkinson's disease; SGL, sub-granular layer; SVZ, sub-ventricular zone; TH, tyrosine hydroxylase; VTA, ventral tegmental area.
\end{abstract}

\title{
Acknowledgements
}

The authors thank MongTien for expert technical assistance in the tissue preparation and immunohistochemical procedures. C.P. is a Viertel Senior Research Fellow. This work was supported byNHMRC project grant \#1042584. The Florey Institute of Neuroscience and Mental Health acknowledges the strong support of the Victorian Government and in particular the funding from the Operational Infrastructure Support Grant. 


\section{References}

Amaral D G, Cowan W M (1980). Subcortical afferents to the hippocampal formation in the monkey.J Comp Neurol, 189(4): 573-591doi:10.1002/cne.901890402 PMID:6769979

Baker S A, Baker K A, Hagg T (2004). Dopaminergic nigrostriatal projections regulate neural precursor proliferation in the adult mouse subventricular zone.Eur J Neurosci, 20(2): 575-579doi:10.1111/j.1460-9568.2004.03486.x PMID:15233767

Ben Abdallah N M, Slomianka L, Vyssotski A L, Lipp H P (2010). Early age-related changes in adult hippocampal neurogenesis in C57 mice.Neurobiol Aging, 31(1): 151-161doi:10.1016/j.neurobiolaging.2008.03.002 PMID:18455269

Bischoff S, Scatton B, Korf J (1979). Biochemical evidence for a transmitter role of dopamine in the rat hippocampus.Brain Res, 165(1): 161-165doi:10.1016/0006-8993(79)90056-8 PMID:218689

Bjorklund A (1978). Monoaminergic inputs to the hippocampus. In: Symposium, C.F. (ed), Functions of the SeptoHippocampal System. Elsevier Excerpta Medica North-Holland, Amsterdam

BjörklundA, DunnettS B (2007). Dopamine neuron systems in the brain: an update.Trends Neurosci, 30(5): 194 202doi:10.1016/j.tins.2007.03.006 PMID:17408759

Borgkvist A, Malmlöf T, Feltmann K, Lindskog M, Schilström B (2012). Dopamine in the hippocampus is cleared by the norepinephrine transporter.Int J Neuropsychopharmacol, 15(4): 531-540 PMID:21669025

Broussard J I, Yang K, Levine A T, Tsetsenis T, Jenson D, Cao F, Garcial, Arenkiel B R, Zhou F M, De Biasi M, Dani J A (2016). Dopamine regulates aversive contextual learning and associated in vivo synaptic plasticity in the hippocampus. Cell Reports, 14(8): 1930-1939doi:10.1016/j.celrep.2016.01.070 PMID:26904943

Carr D B, Sesack S R (2000). GABA-containing neurons in the rat ventral tegmental area project to the prefrontal cortex. Synapse, 38(2): 114-123doi:10.1002/1098-2396(200011)38:2<114::AID-SYN2>3.0.CO;2-R PMID:11018785

Creed M C, Ntamati N R, Tan K R (2014). VTA GABA neurons modulate specific learning behaviors through the control of dopamine and cholinergic systems. Front Behav Neurosci, 8: 8doi:10.3389/fnbeh.2014.00008 PMID:24478655

Drapeau E, Mayo W, Aurousseau C, Le Moal M, Piazza P V, Abrous D N (2003). Spatial memory performances of aged rats in the water maze predict levels of hippocampal neurogenesis. Proc Natl Acad Sci USA, 100(24): 1438514390doi:10.1073/pnas.2334169100 PMID:14614143

Dubois A, Savasta M, Curet O, Scatton B (1986). Autoradiographic distribution of the D1 agonist [3H]SKF 38393, in the rat brain and spinal cord. Comparison with the distribution of D2 dopamine receptors. Neuroscience, 19(1): 125137doi:10.1016/0306-4522(86)90010-2 PMID:2946980

Emre M (2003). Dementia associated with Parkinson's disease. Lancet Neurol, 2(4): 229-237doi:10.1016/S14744422(03)00351-X PMID:12849211

Freundlieb N, François C, Tandé D, Oertel W H, Hirsch E C, Höglinger G U (2006). Dopaminergic substantia nigra neurons project topographically organized to the subventricular zone and stimulate precursor cell proliferation in aged primates. J Neurosci, 26(8): 2321-2325doi:10.1523/JNEUROSCI.4859-05.2006 PMID:16495459

Gasbarri A, Sulli A, Packard M G (1997). The dopaminergic mesencephalic projections to the hippocampal formation in the rat. Prog Neuropsychopharmacol Biol Psychiatry, 21(1): 1-22doi:10.1016/S0278-5846(96)00157-1 PMID:9075256

Gasbarri A, Verney C, Innocenzi R, Campana E, Pacitti C (1994). Mesolimbic dopaminergic neurons innervating the hippocampal formation in the rat: a combined retrograde tracing and immunohistochemical study. Brain Res, 668(1-2): 71-79doi:10.1016/0006-8993(94)90512-6 PMID:7704620

Harley C W (2007). Norepinephrine and the dentate gyrus. Prog Brain Res, 163: 299-318doi:10.1016/S00796123(07)63018-0 PMID: 17765726

Höglinger G U, Rizk P, Muriel M P, Duyckaerts C, Oertel W H, Caille I, Hirsch E C (2004). Dopamine depletion impairs

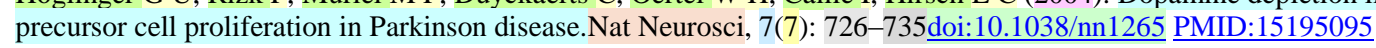

Ito R, Robbins T W, Pennartz C M, Everitt B J (2008). Functional interaction between the hippocampus and nucleus accumbens shell is necessary for the acquisition of appetitive spatial context conditioning. J Neurosci, 28(27): 69506959 doi:10.1523/JNEUROSCI.1615-08.2008 PMID:18596169

Kwon O B, Paredes D, Gonzalez C M, Neddens J, Hernandez L, Vullhorst D, Buonanno A (2008). Neuregulin-1 regulates LTP at CA1 hippocampal synapses through activation of dopamine D4 receptors. Proc Natl Acad Sci USA, 105(40): 15587-15592doi:10.1073/pnas.0805722105 PMID:18832154

Levy G, Schupf N, Tang M X, Cote L J, Louis E D, Mejia H, Stern Y, Marder K (2002). Combined effect of age and severity on the risk of dementia in Parkinson's disease. Ann Neurol, 51(6): 722-729doi:10.1002/ana.10219 PMID: 12112078

Loughlin S E, Foote S L, Bloom F E (1986). Efferent projections of nucleus locus coeruleus: topographic organization of cells of origin demonstrated by three-dimensional reconstruction. Neuroscience, 18(2): 291-306doi:10.1016/0306$\underline{\text { 4522(86)90155-7 } \text { PMID:3736860 }}$ 
Loy R, Koziell D A, Lindsey J D, Moore R Y (1980). Noradrenergic innervation of the adult rat hippocampal formation. J Comp Neurol, 189(4): 699-710 doi:10.1002/cne.901890406 PMID:7381046

Meibach R C, Siegel A (1977). Efferent connections of the hippocampal formation in the rat. Brain Res, 124(2): 197224doi:10.1016/0006-8993(77)90880-0 PMID:402984

Pohle W, Ott T, Müller-Welde P (1984). Identification of neurons of origin providing the dopaminergic innervation of the hippocampus. J Hirnforsch, 25(1): 1-10 PMID:6725937

Regensburger M, Prots I, Winner B (2014). Adult hippocampal neurogenesis in Parkinson's disease: impact on neuronal survival and plasticity. Neural Plast, 2014: 454696doi:10.1155/2014/454696 PMID:25110593

Reymann K, Pohle W, Müller-Welde P, Ott T (1983). Dopaminergic innervation of the hippocampus: evidence for midbrain raphe neurons as the site of origin. Biomed Biochim Acta, 42(10): 1247-1255 PMID:6202300

Rosen Z B, Cheung S, Siegelbaum S A (2015). Midbrain dopamine neurons bidirectionally regulate CA3-CA1 synaptic drive. Nat Neurosci, 18(12): 1763-1771 doi:10.1038/nn.4152 PMID:26523642

Samuels E R, Szabadi E (2008). Functional neuroanatomy of the noradrenergic locus coeruleus: its roles in the regulation of arousal and autonomic function part I: principles of functional organisation. Curr Neuropharmacol, 6(3): 235253doi:10.2174/157015908785777229 PMID:19506723

Sara S J (2009). The locus coeruleus and noradrenergic modulation of cognition. Nat Rev Neurosci, 10(3): 211223doi:10.1038/nrn2573 PMID:19190638

Scatton B, Simon H, Le Moal M, Bischoff S (1980). Origin of dopaminergic innervation of the rat hippocampal formation. Neurosci Lett, 18(2): 125-131 doi:10.1016/0304-3940(80)90314-6 PMID:7052484

Schwab M E, Javoy-Agid F, Agid Y (1978). Labeled wheat germ agglutinin (WGA) as a new, highly sensitive retrograde tracer in the rat brain hippocampal system. Brain Res, 152(1): 145-150doi:10.1016/0006-8993(78)90140-3 PMID:79432

Schwarz L A, Miyamichi K, Gao X J, Beier K T, Weissbourd B, DeLoach K E, Ren J, Ibanes S, Malenka R C, Kremer E J, Luo L (2015). Viral-genetic tracing of the input-output organization of a central noradrenaline circuit. Nature, 524(7563): 88-92doi:10.1038/nature14600 PMID:26131933

Seib D R, Corsini N S, Ellwanger K, Plaas C, Mateos A, Pitzer C, Niehrs C, Celikel T, Martin-Villalba A (2013). Loss of Dickkopf-1 restores neurogenesis in old age and counteracts cognitive decline. Cell Stem Cell, 12(2): 204214doi:10.1016/j.stem.2012.11.010 PMID:23395445

SimonH, Le MoalM, CalasA (1979). Efferents and afferents of the ventral tegmental-A10 region studied after local injection of [3H]leucine and horseradish peroxidase.Brain Res, 178(1): 17-40doi:10.1016/0006-8993(79)90085-4 PMID:91413

Small S A, Schobel S A, Buxton R B, Witter M P, Barnes C A (2011). A pathophysiological framework of hippocampal dysfunction in ageing and disease. Nat Rev Neurosci, 12(10): 585-601doi:10.1038/nrn3085 PMID:21897434

Smith C C, Greene R W (2012). CNS dopamine transmission mediated by noradrenergic innervation. J Neurosci, 32(18): 6072-6080doi:10.1523/JNEUROSCI.6486-11.2012 PMID:22553014

Spalding K L, BergmannO, Alkass K, Bernard S, Salehpour M, Huttner H B, Boström E, Westerlund I, Vial C, Buchholz B A, Possnert G, Mash D C, Druid H, Frisén J (2013). Dynamics of hippocampal neurogenesis in adult humans. Cell, 153(6): 1219-1227 $\underline{\text { doi:10.1016/j.cell.2013.05.002 PMID:23746839 }}$

Sui Y, Horne M K, Stanić D (2012). Reduced proliferation in the adult mouse subventricular zone increases survival of olfactory bulb interneurons. PLoS ONE, 7(2): e31549doi:10.1371/journal.pone.0031549 PMID:22363671

Suzuki K, Okada K, Wakuda T, Shinmura C, Kameno Y, Iwata K, Takahashi T, Suda S, Matsuzaki H, Iwata Y, Hashimoto K, Mori N (2010). Destruction of dopaminergic neurons in the midbrain by 6-hydroxydopamine decreases hippocampal cell proliferation in rats: reversal by fluoxetine. PLoS ONE, 5(2): e9260doi:10.1371/journal.pone.0009260 PMID:20174647

Swanson L W (1982). The projections of the ventral tegmental area and adjacent regions: a combined fluorescent retrograde tracer and immunofluorescence study in the rat. Brain Res Bull, 9(1-6): 321-353doi:10.1016/03619230(82)90145-9 PMID:6816390

Szabadi E (2013). Functional neuroanatomy of the central noradrenergic system. J Psychopharmacol, 27(8): 659693doi:10.1177/0269881113490326 PMID:23761387

Verney C, Baulac M, Berger B, Alvarez C, Vigny A, Helle K B (1985). Morphological evidence for a dopaminergic terminal field in the hippocampal formation of young and adult rat. Neuroscience, 14(4): 1039-1052doi:10.1016/03064522(85)90275-1 PMID:2860616

Wisman L A, Sahin G, Maingay M, Leanza G, Kirik D (2008). Functional convergence of dopaminergic and cholinergic input is critical for hippocampus-dependent working memory. J Neurosci, 28(31): 7797-

7807 doi:10.1523/JNEUROSCI.1885-08.2008 PMID:18667612

Wyss J M, Swanson L W, Cowan W M (1979). A study of subcortical afferents to the hippocampal formation in the rat. Neuroscience, 4(4): 463-476doi:10.1016/0306-4522(79)90124-6 PMID:107474 